\title{
Telemedicine use in 2020 during the COVID-19 pandemic among community dwelling U.S. Medicare beneficiaries
}

\author{
Mary Lynn Davis-Ajami*1 ${ }^{*}$, Zhiqiang K. Lu ${ }^{2}$, Jun $\mathrm{Wu}^{3}$ \\ ${ }^{1}$ Indiana University School of Nursing, United States \\ ${ }^{2}$ University of South Carolina College of Pharmacy, United States \\ ${ }^{3}$ Presbyterian College School of Pharmacy, United States
}

Received: August 25, 2021

DOI: $10.5430 /$ jha.v10n4p32
Accepted: September 21, 2021

URL: https://doi.org/10.5430/jha.v10n4p32

Online Published: September 28, 2021

\begin{abstract}
Objective: CMS reimbursement regulations for telemedicine changed after the onset of the COVID-19 pandemic. This study aimed to assess telemedicine utilization patterns offered by health care providers and used by Medicare beneficiaries during the COVID-19 pandemic during 2020.

Methods: This study used the Fall 2020 Medicare Current Beneficiary Survey (MCBS) supplemental COVID-19 survey to identify Medicare beneficiaries ( $\geq 65$ years) with a regular place for medical care that offered telemedicine during 2020. Major outcomes: prevalence for whether telemedicine was offered before and during the pandemic, telemedicine use, and digital access to telemedicine. Logistic regression identified the demographic factors associated with telemedicine use.

Results: The study sample included 4,380 eligible individual Medicare beneficiaries $\geq 65$ years. Of those, $42.9 \%$ made telemedicine visits during the pandemic. Approximately $60 \%$ of the telemedicine visits were conducted via telephone. Telemedicine was offered to $18 \%$ of the respondents before the pandemic vs. $64 \%$ during year 2020 of the pandemic. Among telemedicine users, $57.2 \%, 28.3 \%$, and $14.5 \%$ used voice calls, video calls, and both voice and video calls for health care appointments, respectively. Overall telemedicine use varied by sex, race, and region. Individuals 65-74 years, female, living in a metropolitan area, with higher incomes were more likely to make video visits. Experience using telecommunications via the internet influenced telemedicine use significantly.

Conclusions: Telemedicine offered to older Medicare beneficiaries increased dramatically after the onset of the COVID-19 pandemic. Yet, less than half used telemedicine and differences in utilization existed by demographic characteristics.
\end{abstract}

Key Words: Coronavirus, COVID-19, Telemedicine, Medicare

\section{INTRODUCTION}

The Centers for Medicare \& Medicaid Services (CMS) expanded telemedicine coverage and reimbursement in March, and again in April, 2020 in response to the COVID-19 pandemic in the US. CMS described 3 broad telemedicine vir- tual service classifications: 1) telehealth, 2) virtual check ins, and 3) E-visits. Virtual check ins differ from telehealth by their brevity and ability to be conducted via phone or by an exchange of a video or image. E-visits use online patient portals for established patients. CMS defined telehealth as a

\footnotetext{
*Correspondence: Mary Lynn Davis-Ajami; Email: mdavisaj@iu.edu; Address: Department of Science of Nursing Care, Indiana University School of Nursing, 600 Barnhill Drive, Indianapolis, IN 46202, United States.
} 
real-time, 2-way communication that uses telecommunications technology with audio and visual capabilities. ${ }^{[1]}$ The CMS emergency action added 80 services accessible via interactive audio-visual apps, or by phone. Under these new regulations health care providers could bill at the same rate as face-to-face encounters for new and established patients and across state lines for states with modified licensure requirements, for office, hospital, inpatient rehabilitation, hospice, and home health services. ${ }^{[2,3]}$ Other payers may allow telehealth via a telephone without video, but CMS requires audio and visual capabilities when billing Medicare. ${ }^{[4]}$ Early in the pandemic Medicare policy evolved to include guidance for fee-for-service, Medicare Advantage, other existing telehealth policies, and other technology-enabled services. ${ }^{[5]}$ Medicare also gave any health care provider eligible to bill Medicare irrespective of their location and the ability to bill for telehealth. ${ }^{[3,6]}$ CMS also waived regulations for real-time platforms, resulting in more technologies approved for use (e.g., Zoom, FaceTime, Google Hangouts, etc.). ${ }^{[4]}$

However, pre-existing issues, e.g., internet access, broadband speed, end user technological savvy, and demographic disparities, raised concerns for disparate uptake in telemedicine use among elders during the pandemic. ${ }^{[7,8]}$ One study showed that $38 \%$ of older people lacked technology experience and were not ready for video visits. ${ }^{[7]}$ Those $>75$ years, male, unmarried, black or Hispanic, less educated, lower income, and from a nonmetropolitan area were more likely to show telemedicine unreadiness. ${ }^{[7]}$ Sensory motor and cognitive declines common among older individuals may contribute to telemedicine unreadiness. Physical, structural, and knowledge base attributes may influence the adoption of telemedicine in elders and may add to the maldistribution of health care services during the pandemic. ${ }^{[7]}$

Infrastructure and technology disparities exist across US geographical regions in that may present barriers to telemedicine use among older Americans. ${ }^{[8]}$ A 2021 survey showed, 61\% of adults ( $\geq 65$ years) own smartphones, but only $43 \%$ own phones if $\geq 75$ years. $^{[9]}$ Seventy-seven percent of older adults subscribed to home broadband. ${ }^{[9]}$ However, smartphone ownership and broadband adoption vary by household income and education level, and disparities in broadband access by residential location and race persist. ${ }^{[9]}$ Moreover, $41 \%$ of older adults had little to no online experience. ${ }^{[10]}$ Yet, since COVID-19 reduced regulatory barriers, our understanding of how that change affected telemedicine usage in Medicare beneficiaries is limited.

To understand how the COVID-19 pandemic affected health care access in Medicare beneficiaries, CMS leveraged the Medicare Current Beneficiary Survey (MCBS) panel de- sign to administer rapid response surveys to supplement the main MCBS in Summer and Fall of 2020. ${ }^{[11]}$ New questions were added to gather information about telemedicine use. Since CMS telemedicine reimbursement policy changed relatively recently, the literature related to Medicare beneficiary telemedicine use during the COVID-19 pandemic continues to evolve. Our knowledge about whether health care providers offered telemedicine more frequently than before the onset of COVID-19, and the extent of changes in telemedicine utilization patterns in community-welling older Medicare beneficiaries, is formative. The overarching purpose of this study was to explore telemedicine use patterns among community dwelling older Medicare beneficiaries during year 2020 of the COVID-19 pandemic. The study had the following specific objectives: to 1) examine pandemic-related telemedicine prevalence offered by health care providers prior to and during the COVID-19 pandemic among Medicare beneficiaries who reported a regular place for medical care and 2) explore whether community-dwelling Medicare beneficiaries with a regular medical provider made telehealth medical appointments when offered that alternative, and 3) to determine the type of telemedicine services used by those who made telemedicine appointments.

\section{Methods}

\subsection{Data source}

The MCBS, sponsored by CMS, gives information about health care use, access, expenditures, and factors affecting health care utilization in a representative national sample of the Medicare population. ${ }^{[12]}$ In response to the COVID-19 pandemic outbreak, CMS prepared a series of rapid response surveys to supplement the main MCBS. The first two supplements were administered in Summer and Fall 2020 to assess and understand the impact of the COVID-19 pandemic on Medicare beneficiaries. This study used the Fall 2020 supplement public use file to examine how community-dwelling Medicare beneficiaries used telemedicine for their appointments during the COVID-19 pandemic.

\subsection{Study sample}

MCBS respondents $\geq 65$ years with an established place for medical care were included (see Figure 1). This group was further restricted to only include those offered telemedicine after the onset of COVID-19 to form the primary study sample measuring telemedicine use and associated outcomes.

\subsection{Outcome measures}

Telemedicine offered during the pandemic was measured using responses to the question, "Does your usual provider offer telephone or video appointments, so that you don't need to physically visit their office or facility?" If yes, then the type 
of telemedicine offered during the pandemic was measured. Next, the telemedicine offered before the COVID-19 pandemic was defined from this query, "Did your usual provider offer telephone or video appointments before the COVID-19 pandemic?". If yes, then the type of telemedicine offered before the pandemic was measured using the response to the question "Did they offer telephone appointments, video appointments, or both?". Finally, the impact of COVID-19 pandemic on the regularly scheduled appointments was measured using the question, "Since July 1, 2020, did your usual provider offer you a telephone or video appointment to replace a regularly scheduled appointment?" If yes, then the type of telemedicine offered as an alternative appointment was measured.

Among respondents offered telemedicine during the pandemic, overall telemedicine use, and the type of telemedicine use for appointments were measured. Overall telemedicine use was defined by responses to the question, "Since July 1 , 2020, have you had an appointment with a doctor or other health professional by telephone or video?" If yes, then the type of telemedicine use was measured based on response to the next question, "Was it a telephone appointment, video appointment, or both?" The type of telemedicine use was then collapsed into video and non-video appointments for analysis. Video appointment was defined if respondents used video only or both video and telephone for appointments. Non-video appointments were defined as a telephone call only.

\subsection{Digital access to telemedicine}

Access to telemedicine was measured by three elements: 1) digital device owned (i.e., computer, smartphone, tablet), 2) access to internet, and 3) previous experience of making video or voice calls over the internet (e.g., Zoom, Skype, and FaceTime).

\subsection{Characteristics of study sample}

Demographic variables included age (65-74 and 75+ years), sex, race, metropolitan residence designated by core-based statistical area (metro and non-metro), census region (Northeast, Midwest, South, and West), income group $(<\$ 25,000$ and $\$ 25,000+$ ), Medicare - Medicaid dual eligibility, and Medicare Advantage coverage. Chronic conditions were identified from the survey responses. The MCBS uses a chronic condition classification system from the US Department of Health and Human Services (HHS) Office of the Assistant Secretary of Health (OASH) used in five HHS data systems, including CMS. ${ }^{[13]}$ The number of chronic conditions were collapsed into $0-1,2-3$, and 4+ categorical variables.

\subsection{Data analysis}

MCBS used a complex survey design. The sampling and replicate weights created in the data files were applied to all analyses to obtain national estimates.

\subsubsection{Characteristics of study sample and subgroup of telemedicine users}

Weighted relative frequencies described the characteristics of the study sample. Chi-square tests were used to compare the characteristics of telemedicine users vs. no telemedicine users. For those who reported telemedicine use, weighted relative frequencies and described this subgroup characteristics by the type of telemedicine use for appointments (video vs. phone). Chi-square tests were used to compare the characteristics of those using video vs. phone for telemedicine visits.

\subsubsection{Digital device owned, internet access, and experi- ence with internet use}

The distributions of the type of digital device owned (i.e., computer, smart phone, and tablet), internet access, and previous experience of video or voice call via internet were described by weighted relative frequencies compared by chisquare tests in the study sample (telemedicine users vs. nontelemedicine users) and in the subgroup of telemedicine users (video call users vs. voice call users).

\subsubsection{Telemedicine offered during pandemic vs. before pandemic}

Telemedicine offered by providers and awareness of telemedicine offered during the pandemic vs. before the pandemic was described by weighted relative frequencies and compared by chi-square tests. The type of telemedicine offered (i.e., phone, video, or both) was also described and compared during pandemic vs. before pandemic.

\subsubsection{Respondent characteristics associated with overall telemedicine use and type of telemedicine use}

Two logistic regression models were used to identify significant demographic and health characteristics associated with overall telemedicine use and video call use. The first logistic regression model included all participants in the study sample. The dependent variable was overall telemedicine use (Yes/No). The second logistic regression model included a subgroup of those who used telemedicine during the pandemic. The dependent variable was video call (Yes/No). The demographic and health characteristics included in the logistic regression models were age, sex, race, metropolitan residence, region, income, Medicare-Medicare dual eligibility, Medicare Advantage coverage, and number of chronic conditions.

Statistical significance was set at $p<.05$. Analyses were 
performed using SAS, version 9.4 (SAS Institute, Inc., Cary, NC). This study was granted non-human subjects research status.

\section{Results}

\subsection{Overall telemedicine use}

Among 9,686 Medicare beneficiaries participating in the MCBS Fall COVID-19 survey, 4,380 eligible older individuals reported being offered telemedicine by their health care providers during the COVID-19 pandemic, representing 26.8 million Medicare beneficiaries (see Figure 1). During the pandemic, $42.9 \%$ of our study sample used telemedicine for their appointments, in which $57.2 \%, 28.3 \%$, and $14.5 \%$ used voice calls, video calls, and both for appointments, respec- tively. Nearly $50 \%$ of respondents were offered telemedicine to replace their regularly scheduled appointments during the pandemic.

\subsection{Characteristics of study sample by telemedicine use and subgroup of telemedicine use (video vs. phone)}

In Table 1, telemedicine users showed higher proportions of males, non-white, Medicare-Medicaid dual eligibility, and four or more chronic conditions than non-telemedicine users. Among those who used telemedicine for their appointments (see Table 2), respondents using video calls showed higher proportions of age (65-74 years) and metropolitan residence but lower proportions of income $(<\$ 25,000)$, MedicareMedicaid dual eligibility, and Medicare Advantage than those using phone calls.

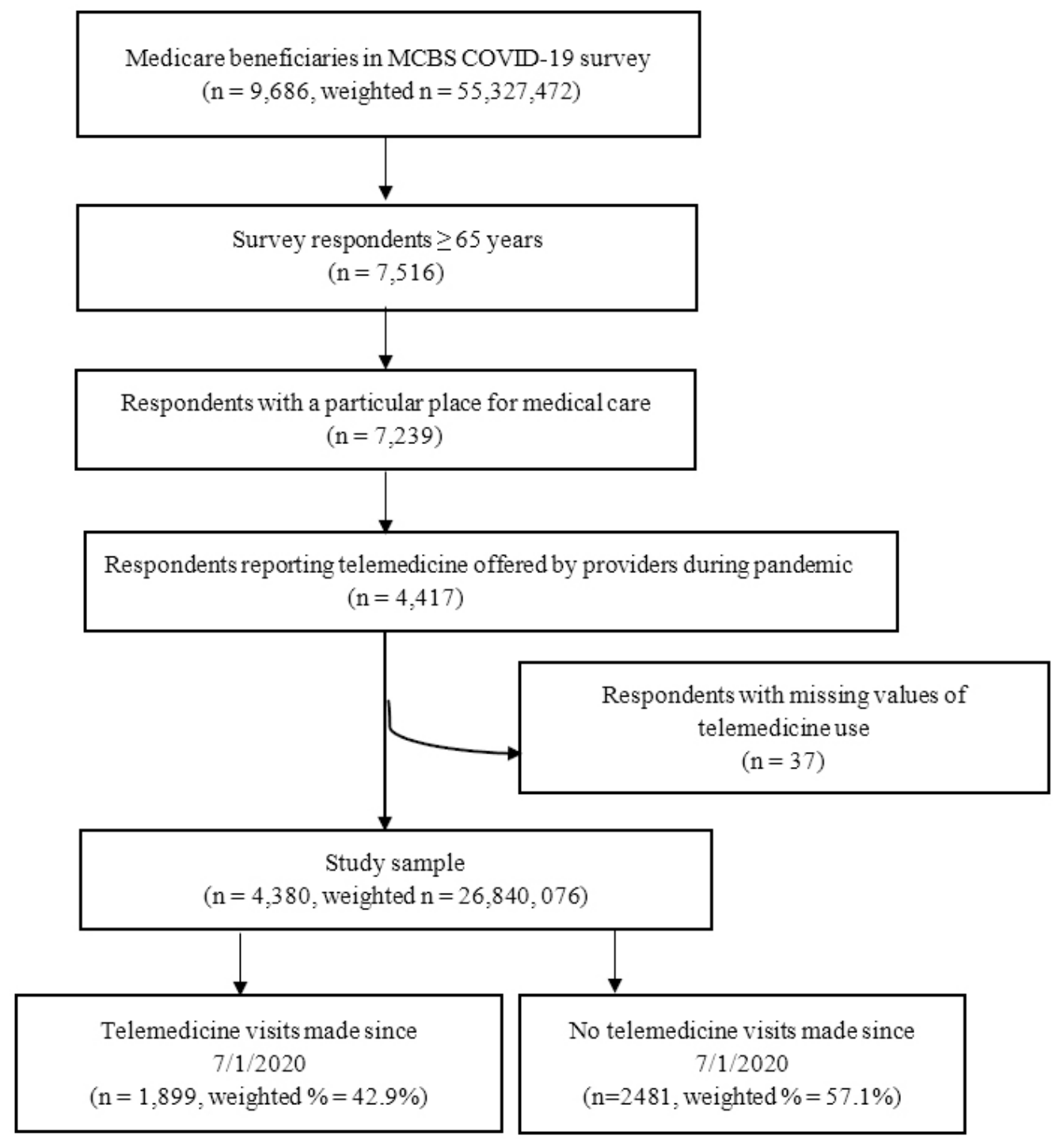

Figure 1. Study sample 
3.3 Digital device owned, internet access, and experi- only $(p<.001)$.

ence of internet use by telemedicine use and video call use

Figure 2 displays no differences in owning electronic devices and access to the Internet between telemedicine and non-telemedicine users. However, the proportion of previous experience of making video or voice calls over the internet in telemedicine users was $57.3 \%$ vs. $45.3 \%$ in non-telemedicine users $(p<.001)$. Telemedicine users using video calls for appointments reported higher proportions owning electronic devices, having internet access, and previous experience making internet video or voice calls than those using voice calls

\subsection{Patterns of telemedicine offered by providers (before vs. during pandemic)}

Figure 3 compared telemedicine offered before and during the pandemic. Providers offered telemedicine to $18 \%$ of the MCBS respondents before vs. $64 \%$ during the pandemic. Before the pandemic, $31 \%$ did not know whether providers offered telemedicine vs. $23 \%$ during the pandemic. Among those offered telemedicine, both voice and video calls were offered to $45 \%$ of the respondents before vs. $60.2 \%$ during the pandemic.

Table 1. Characteristics of older community dwelling US Medicare beneficiaries by telemedicine visit or not during COVID-19 $(\mathrm{n}=4,380)$

\begin{tabular}{|c|c|c|c|c|}
\hline Variable & $\begin{array}{l}\text { All }(n=4,380) \text {, } \\
\text { weighted \% } \\
(95 \% \text { CI })\end{array}$ & $\begin{array}{l}\text { Telemedicine } \\
(\mathrm{n}=1,899) \text {, } \\
\text { weighted \% } \\
(95 \% \mathrm{CI})\end{array}$ & $\begin{array}{l}\text { No telemedicine } \\
(\mathrm{n}=\mathbf{2 , 4 8 1}), \\
\text { weighted \% } \\
(\mathbf{9 5 \%} \mathrm{CI})\end{array}$ & $p$-value \\
\hline Age (years) & & & & .53 \\
\hline $65-74$ & $63.4(62.0,64.8)$ & $62.8(60.4,65.3)$ & $63.8(62.0,65.7)$ & \\
\hline $75+$ & $36.6(35.2,38.0)$ & $37.2(34.7,39.6)$ & $36.2(34.3,38.0)$ & \\
\hline Sex, male & $44.3(42.5,46.1)$ & $46.7(44.1,49.3)$ & $42.5(40.1,44.9)$ & .02 \\
\hline Race & & & & .004 \\
\hline White non-Hispanic & $77.9(75.6,80.1)$ & $75.0(72.0,77.9)$ & $80.2(77.8,82.5)$ & \\
\hline Black non-Hispanic & $8.2(6.8,9.5)$ & $9.7(7.8,11.7)$ & $7.0(5.5,8.6)$ & \\
\hline Hispanic & $8.3(6.7,10.0)$ & $9.8(7.7,11.9)$ & $7.3(5.4,9.1)$ & \\
\hline Other & $5.5(4.6,6.4)$ & $5.5(4.3,6.7)$ & $5.5(4.3,6.8)$ & \\
\hline Metropolitan Residence & $84.7(83.0,86.4)$ & $85.5(83.1,88.0)$ & $84.1(82.1,86.1)$ & .31 \\
\hline Region & & & & .001 \\
\hline Northeast & $18.0(16.3,19.7)$ & $16.0(13.3,18.8)$ & $19.5(17.5,21.6)$ & \\
\hline Midwest & $21.1(18.5,23.8)$ & $18.4(14.9,21.9)$ & $23.2(20.3,26.1)$ & \\
\hline South & $35.7(33.4,38.0)$ & $37.9(34.5,41.3)$ & $34.1(31.7,36.6)$ & \\
\hline West & $25.1(22.9,27.3)$ & $27.7(24.3,31.0)$ & $23.2(20.9,25.5)$ & \\
\hline Income $(<\$ 25,000)$ & $21.9(20.2,23.5)$ & $23.1(20.8,25.5)$ & $20.9(18.9,22.9)$ & .12 \\
\hline Medicare-Medicaid dual eligibility & $8.5(7.4,9.5)$ & $10.3(8.7,12.0)$ & $7.1(6.0,8.2)$ & .001 \\
\hline Medicare advantage & $40.7(38.4,43.0)$ & $41.4(38.2,44.6)$ & $40.2(37.4,43.1)$ & .56 \\
\hline Number of chronic conditions & & & & $<.001$ \\
\hline $0-1$ & $16.1(14.5,17.7)$ & $12.1(10.3,13.7)$ & $19.1(17.0,21.4)$ & \\
\hline $2-3$ & $38.6(37.0,40.2)$ & $34.8(32.3,37.3)$ & $41.4(39.1,43.7)$ & \\
\hline $4+$ & $45.3(43.6,47.1)$ & $53.2(50.6,55.8)$ & $39.5(36.9,42.1)$ & \\
\hline
\end{tabular}


Table 2. Characteristics of community dwelling older Medicare beneficiaries using telemedicine (video vs. phone calls)

\begin{tabular}{|c|c|c|c|}
\hline Variable & $\begin{array}{l}\text { Video }(n=1,142), \text { weighted } \\
\%(95 \% \text { CI })\end{array}$ & $\begin{array}{l}\text { Phone }(\mathrm{n}=757), \text { weighted \% } \\
(95 \% \text { CI })\end{array}$ & $p$-value \\
\hline Age (years) & & & $<.001$ \\
\hline $65-74$ & $69.7(66.3,73.2)$ & $57.6(54.4,60.8)$ & \\
\hline $75+$ & $30.3(26.8,33.7)$ & $42.4(39.2,45.6)$ & \\
\hline Sex, male & $44.2(39.8,48.5)$ & $48.6(45.3,51.9)$ & .12 \\
\hline Race & & & .05 \\
\hline White non-Hispanic & $78.4(74.8,81.9)$ & $72.5(68.6,76.4)$ & \\
\hline Black non-Hispanic & $8.5(6.0,10.9)$ & $10.7(7.9,13.5)$ & \\
\hline Hispanic & $7.5(5.7,9.3)$ & $11.4(8.4,14.4)$ & \\
\hline Other & $5.7(3.6,7.8)$ & $5.4(3.9,6.9)$ & \\
\hline Metropolitan Residence & $88.3(85.2,91,5)$ & $83.5(80.2,86.8)$ & .02 \\
\hline Region & & & .48 \\
\hline Northeast & $17.7(12.9,22.4)$ & $14.9(12.6,17.1)$ & \\
\hline Midwest & $16.9(12.5,21.2)$ & $19.6(15.6,23.6)$ & \\
\hline South & $38.1(32.1,44.1)$ & $37.6(33.9,41.3)$ & \\
\hline West & $27.4(23.4,31.4)$ & $27.9(23.9,32.0)$ & \\
\hline Income $(<\$ 25,000)$ & $16.6(13.9,19.3)$ & $28.0(24.9,31.1)$ & $<.001$ \\
\hline Medicare-Medicaid dual eligibility & $6.6(5.0,8.2)$ & $13.0(10.7,15.2)$ & $<.001$ \\
\hline Medicare advantage & $36.8(32.5,41.0)$ & $44.9(41.1,48.5)$ & .001 \\
\hline Number of chronic conditions & & & .73 \\
\hline $0-1$ & $12.4(9.6,15.1)$ & $11.8(9.5,14.0)$ & \\
\hline $2-3$ & $35.8(31.8,39.7)$ & $34.2(30.7,37.7)$ & \\
\hline $4+$ & $51.9(48.3,55.5)$ & $54.0(50.4,57.6)$ & \\
\hline
\end{tabular}

\subsection{Respondent characteristics associated with overall telemedicine use and video call use}

Logistic regression results indicate that sex, race, region, and the number of chronic conditions were associated with overall telemedicine use during the pandemic (see Table 3). Medicare beneficiaries being male (vs. female), black (vs. white), residents in South or West (vs. Northeast), and having multiple chronic conditions were more likely to use telemedicine $(p<.05)$. The type of telemedicine used (video vs. non video) was associated with age, sex, residence, income, and Medicare Advantage coverage. Telemedicine users being 65-74 years (vs. 75 years), female (vs. male) and metropolitan residents, and reporting income $\geq \$ 25,000$ (vs. $<\$ 25,000)$ were more likely to use video call for telemedicine appointments $(p<.05)$.

\section{Discussion}

Early in the COVID-19 outbreak, CMS broadened the types of services patients could receive through telemedicine. Prior to the expansion of telemedicine services, our study found only $18 \%$ of MCBS respondents were offered telemedicine as a means to see their provider for a health care visit. After the CMS guidelines changed, $64 \%$ of MCBS respondents were offered provider services via telemedicine. This shows a large increase in the proportions of patients being offered telemedicine compared to before the COVID-19 outbreak. This increase may reflect how quickly health care providers responded to lockdown and social distancing measures to provide alternatives to in-person medical appointments and health systems adjusted their capacity to provide telemedicine. Another study using commercial insurance 
data showed one-third of provider visits were conducted via telemedicine early on during the COVID-19 pandemic. ${ }^{[14]} \mathrm{A}$ different research study looking at an academic health system also found a tremendous increase in provider visits conducted via telemedicine rather than in person after the CMS guidelines broadened. The academic health system in that study only showed $0.5 \%$ of visits were conducted via telemedicine before COVID-19 vs. $41.2 \%$ during the pandemic. ${ }^{[15]} \mathrm{Be}-$ fore the pandemic one-third of Medicare beneficiaries in our study were not aware if providers offered telemedicine. Although this decreased to less than one-fourth during the pandemic, this shows a persistent lack of awareness about available telemedicine services after the onset of COVID-
19. During non-pandemic times Medicare beneficiaries may not need or want telemedicine services as a replacement for in-person medical appointments. However, during the emergence of COVID-19 and subsequent restrictions on normal activity, telemedicine became a vital link to medical care. All respondents in our study had a regular place for medical care. However, the MCBS did not assess their awareness of the CMS telemedicine policy change enacted after the pandemic outbreak or how well health care providers informed their patients about the availability of telemedicine visits. Nevertheless, patient awareness increased, although approximately one quarter of patients remained unaware of the telemedicine services offered by their regular medical provider.
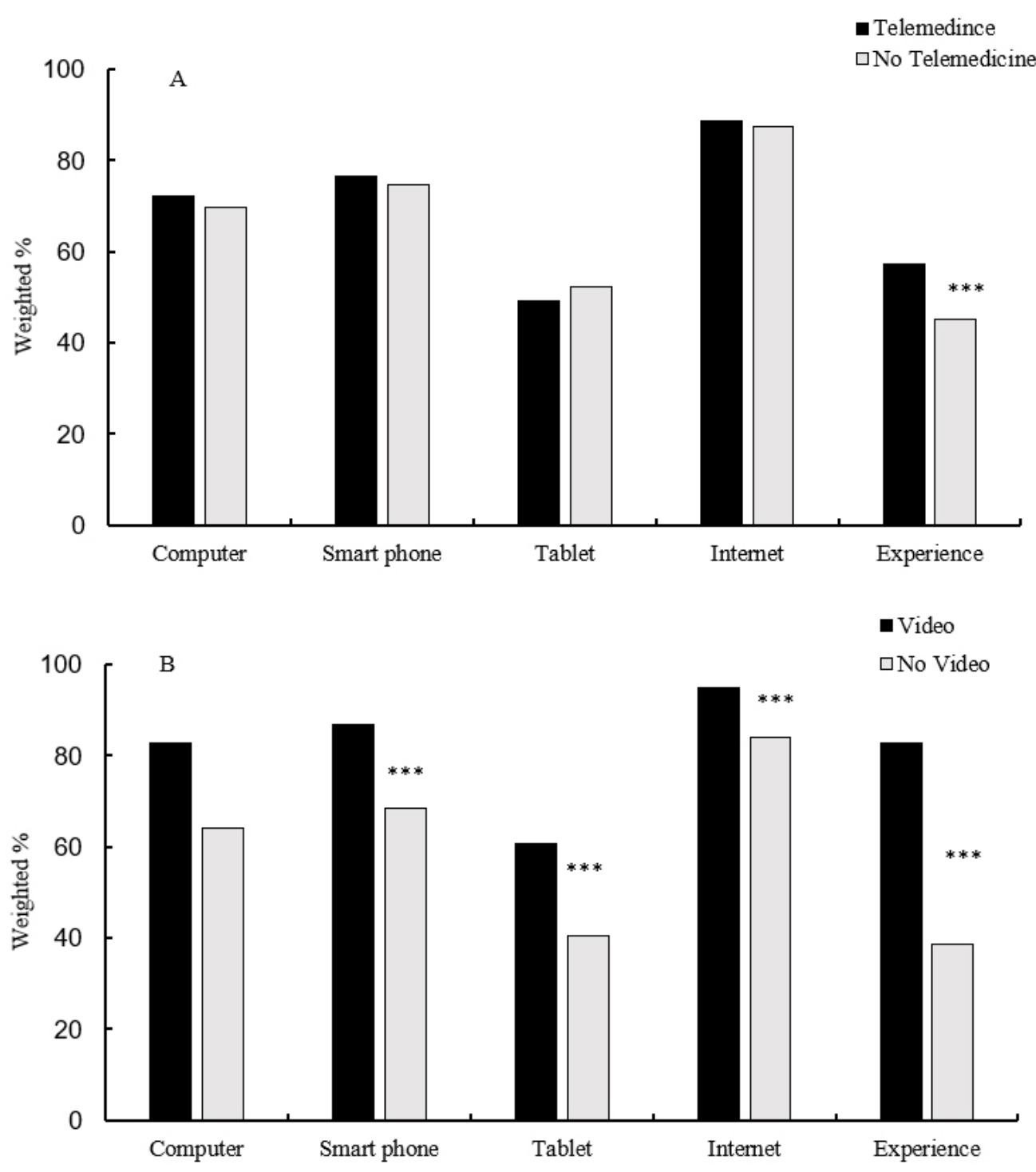

Figure 2. Digital device owned, access to internet, and experience of video or voice call via internet by overall telemedicine use (A) and video call use (B) in older community dwelling Medicare beneficiaries during COVID-19 (***p $<.001)$ 


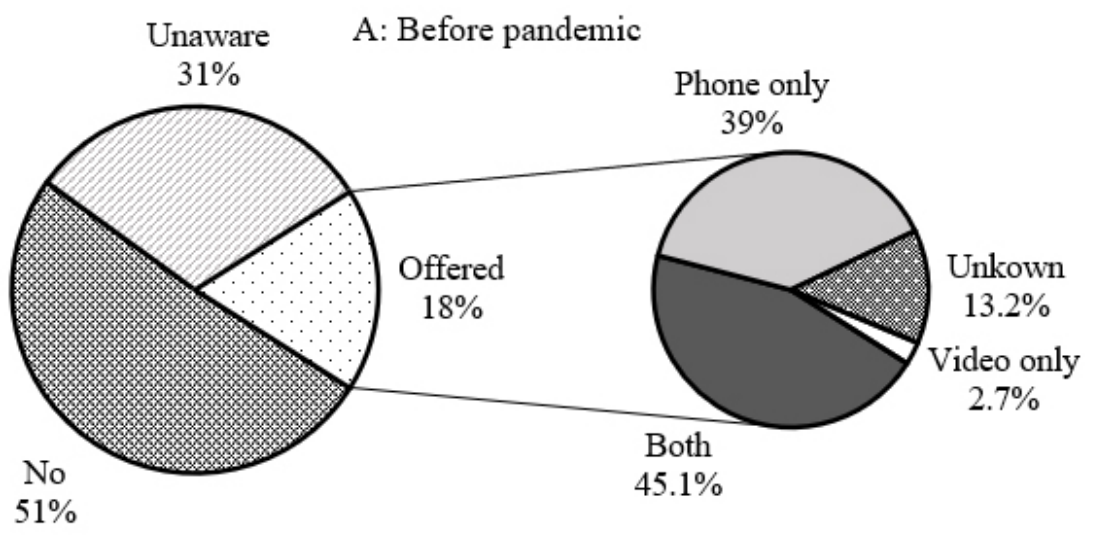

B: During pandemic

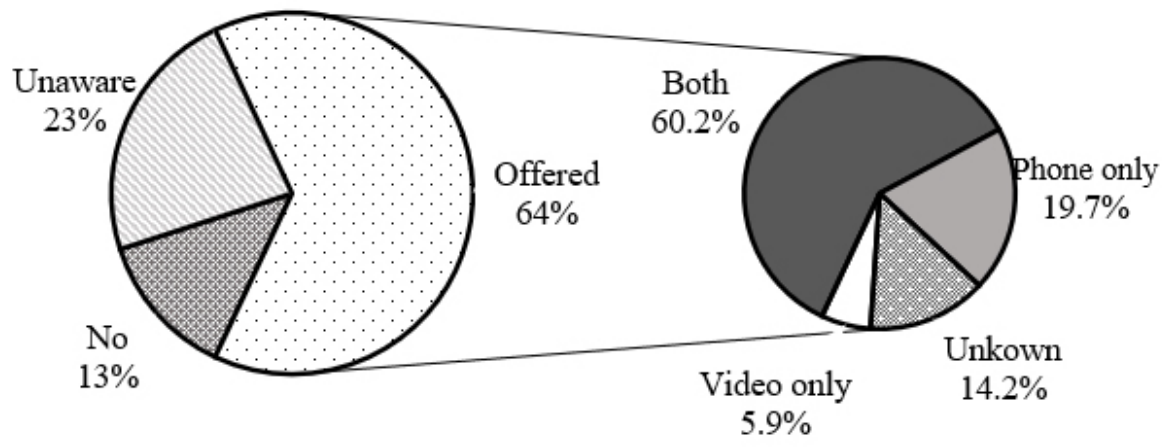

Figure 3. Digital device owned, access to internet, and experience of video or voice call via internet by overall telemedicine use (A) and video call use (B) in older community dwelling Medicare beneficiaries during COVID-19
Overall telemedicine use (video or telephone calls) showed different racial disparity trends from previous studies. Our study showed black Medicare beneficiaries were $46 \%$ more likely to use telemedicine than whites $(\mathrm{OR}=1.46,95 \%$ CI: 1.10-2.00). In contrast, Drake et al.'s study reported black patients $10 \%$ less likely than those of white race to use telemedicine (OR $=0.90,95 \%$ CI: 0.88-0.91). ${ }^{[16]}$ Kakani et al.'s study showed no significant difference in telemedicine use between black and white patients $(\mathrm{OR}=0.98,95 \% \mathrm{CI}$ : 0.92-1.04). ${ }^{[15]}$ Although the Drake and Kakani studies used academic health center encounter data, the studies were conducted in geographically different regions of the country, North Carolina and California, respectively. The Drake study looked at office visits across all insurance providers and age groups where just a little more than one third of those visits were among those covered by Medicare. Their results may not fully reflect the characteristics of the Medicare population.

Our study queried patients directly about telemedicine and is representative of the older Medicare beneficiary population. Telemedicine is a broad term encompassing telehealth (real-time two-way phone, video, or both), ${ }^{[17]}$ virtual check ins, and E-visits. The MCBS survey questions used for our study fit the definition of telehealth. Virtual check-ins use phone or integrated audio/video systems, but limit encounters from the time of the last visit (not within 7 days) and by the time to the next visit (cannot lead to another visit within 24 hours). ${ }^{[18]}$ E-visits use online or digital patient portals to conduct non-face-to-face medical encounters. ${ }^{[19]}$ Telemedicine is the term used in other studies, and our study could not differentiate telehealth from virtual check ins. The CMS questions do not provide information about the time span between appointments, or if visits were conducted through a patient portal.

Our study found the following regional variations: the US Southern and Western regions were more likely to use telemedicine than the Northeast. One study using commercial claims data found greater likelihood of lower telemedicine use during the pandemic in the counties with lower income, lower population density, and fewer COVID19 cases per capita at the beginning of the pandemic. ${ }^{[14]}$ Although our study showed regional differences, we could not assess population density, COVID-19 case numbers, differences in the implementation and adherence to lockdown measures or social distancing, or health system telemedicine capacity among others. 
Table 3. Characteristics associated with telemedicine use in community dwelling older Medicare beneficiaries

\begin{tabular}{|c|c|c|}
\hline \multirow{2}{*}{ Independent variable } & \multicolumn{2}{|c|}{ Odds ratio (95\% CI) } \\
\hline & Overall telemedicine use $(n=4,380)$ & Video call use $(n=1,899)$ \\
\hline \multicolumn{3}{|l|}{ Age (years) } \\
\hline $65-74$ & 1.00 & 1.00 \\
\hline $75+$ & $0.99(0.86,1.15)$ & $0.57(0.47,0.70)$ \\
\hline \multicolumn{3}{|l|}{ Sex } \\
\hline Male & 1.00 & 1.00 \\
\hline Female & $0.82(0.71,0.95)$ & $1.40(1.09,1.78)$ \\
\hline \multicolumn{3}{|l|}{ Race } \\
\hline White non-Hispanic & 1.00 & 1.00 \\
\hline Black non-Hispanic & $1.46(1.10,2.00)$ & $0.72(0.43,1.20)$ \\
\hline Hispanic & $1.21(0.85,1.74)$ & $0.78(0.52,1.15)$ \\
\hline Other & $0.97(0.69,1.36)$ & $1.18(0.68,2.05)$ \\
\hline Metropolitan Residence & $1.06(0.84,1.35)$ & $1.60(1.10,2.34)$ \\
\hline \multicolumn{3}{|l|}{ Region } \\
\hline Northeast & 1.00 & 1.00 \\
\hline Midwest & $0.99(0.71,1.36)$ & $0.70(0.46,1.05)$ \\
\hline South & $1.34(1.02,1.75)$ & $0.86(0.58,1.26)$ \\
\hline West & $1.43(1.05,1.94)$ & $0.82(0.60,1.14)$ \\
\hline \multicolumn{3}{|l|}{ Income $(<\$ 25,000)$} \\
\hline$<\$ 25,000$ & 1.00 & 1.00 \\
\hline$\geq \$ 25,000$ & $1.10(0.90,1.35)$ & $1.58(1.16,2.16)$ \\
\hline Medicare-Medicaid dual eligibility & $1.30(0.95,1.78)$ & $0.72(0.47,1.18)$ \\
\hline Medicare advantage & $0.99(0.83,1.18)$ & $0.78(0.62,0.98)$ \\
\hline \multicolumn{3}{|l|}{ Number of chronic conditions } \\
\hline $0-1$ & 1.00 & 1.00 \\
\hline $2-3$ & $1.38(1.10,1.73)$ & $0.98(0.64,1.50)$ \\
\hline $4+$ & $2.19(1.76,2.73)$ & $1.02(0.72,1.45)$ \\
\hline
\end{tabular}

The type of telemedicine used during appointments was associated with age, sex, income, and Medicare Advantage coverage. Respondents older than 75 years were less likely to use video calls but being female, living in metropolitan areas, and an income $\geq \$ 25,000$ increased the likelihood of using video calls. Lam et al. found the prevalence of unreadiness for video visits higher in more senior, low-income, and rural area patients, particularly if older than 85 years. ${ }^{[7]}$ Telephone visits offered by providers may enhance telemedicine access for the older population and help give providers the opportunity to conduct visual assessment. ${ }^{[20]}$ Future studies could compare the quality of care and patient engagement between video and telephone visits in the older population. ${ }^{[21,22]}$

Access to telemedicine in older populations may be influenced by electronic device ownership, broadband access, and digital literacy. ${ }^{[8,22,23]}$ Our study showed that most health care providers offered both telephone and video calls for telemedicine visits yet $57 \%$ of MCBS respondents only used telephone calls. Our study found no differences in owning electronic devices and access to the internet between overall telemedicine and non-telemedicine users. However, differences existed in digital access by type of telemedicine use. Owning electronic devices and accessing the internet increased the likelihood a video call appointment. Previous experience in making internet video or voice calls increased the likelihood of overall telemedicine use and video call use for appointments. This finding suggests that older people may need technical help with telemedicine appointments. ${ }^{[8]}$

Our study found respondents with multiple chronic conditions, and particularly those with a greater number of 
chronic conditions, were more likely to use telemedicine. Telemedicine shows promise for chronic disease management to improve treatment outcomes, ${ }^{[24,25]}$ yet information about how telemedicine use during the pandemic influences chronic disease health outcomes and medical costs is limited. Patients with multiple chronic conditions require frequent follow up medical visits. Telemedicine may show direct and indirect cost savings, e.g., transportation, productivity loss, or treatment savings for repeated appointments. ${ }^{[26,27]}$ Economic studies could explore how telemedicine influences health care utilization and associated costs in patients with multiple chronic conditions from different perspectives in both COVID-19 and non-COVID-19 times.

Several limitations should be considered when interpreting the results. The MCBS COVID-19 survey was supplemental to the main survey and focused on COVID-19 impacts on health care use since July 2020 . The results only reflect telemedicine use patterns during summer of 2020. This snapshot may not represent the full pandemic trends over time. Second, the MCBS supplemental telemedicine use only targeted those with a regular place to go for medical care who were offered telemedicine provider visits. The public use file only included community-dwelling beneficiaries. This limits the generalizability of the results. Third, the MCBS
COVID-19 survey public use file did not measure the reasons for telemedicine visits and the visit location, e.g., office, outpatient, or urgent care etc. This hinders interpretations about telemedicine in different settings. Additionally, this study did not assess access to care, internet service quality, or the pervasiveness or understanding of marketing channels or materials explaining changes in telemedicine services.

\section{Conclusions}

Telemedicine offered to older Medicare beneficiaries increased dramatically since the COVID-19 pandemic outbreak. Offering both telephone and video calls expanded access to telemedicine for older patients. Demographic disparities differed in overall and type of telemedicine use. Further research is needed to understand barriers to using telemedicine in Medicare beneficiaries, and the sustainability of the CMS policy changes supporting telemedicine after the pandemic.

\section{FUNDING}

The authors have no funding source to report.

\section{CONFlicts OF INTEREST Disclosure}

The authors declare they have no conflicts of interest.

\section{REFERENCES}

[1] Chan AH. Logistics of Rehabilitation Telehealth: Documentation, Reimbursement, and Health Insurance Portability and Accountability Act. Phys Med Rehabil Clin N Am. 2021; 32: 429-36. PMid: 33814067. https://doi.org/10.1016/j.pmr.2021.01.006

[2] CMS.gov: Billing for Professional Telehealth Distant Site Services During the Public Health Emergency [Internet]. Baltimore: Centers for Medicare and Medicaid Services; [cited 2020 April 3]. Available from: https://www.cms.gov/outreach-and-educationoutr eachffsprovpartprogprovider-partnership-email-arc hive/2020-04-03-mlnc-se

[3] Mediciare telemedicine health care provider fact sheet. [Internet]. Baltimore (MD): Centers for Medicare and Medicaid Services (US) [cited 2020 Mar 17]. Available from: https://www.cms.gov/newsroom/fact-sheets/medicare -telemedicine-health-care-provider-fact-sheet

[4] Lunt S. Telehealth billing during the COVID-19 national emergency. [Internet]. Denver (CO): Medical Group management Association. [cited 2020 Apr 24]. Available from: https://www.mgma.com/resources/revenue-cycle/teleh ealth-billing-during-the-covid-19-national-em

[5] CCHP: Telehealth Coverage Policies in the time of COVID-19. [Internet]. Sacramento (CA): The National Telehealth Poicy Resource Center, Center for Connect Health Policy (US). [cited 2021 Mar 21]. Available from: https://www.cchpca.org/resources/covid -19-telehealth-coverage-policies/

Published by Sciedu Press
[6] Billing for telehealth during COVID-19. [Internet]. Washington DC: Health Resources \& Services Administration (US). [cited 2021 April 24]. Available from: https://telehealth.hhs.gov/provide rs/billing-and-reimbursement/

[7] Lam K, Lu AD, Shi Y, et al. Assessing telemedicine unreadiness among older adults in the United States during the COVID-19 pandemic. JAMA Intern Med. 2020; 180: 1389-91. PMid: 32744593. https://doi.org/10.1001/jamainternmed.2020.2671

[8] Woodall T, Ramage M, LaBruyere JT, et al. Telemedicine Services During COVID-19: Considerations for Medically Underserved Populations. J Rural Health. 2021; 37: 231-4. PMid: 32613657. https://doi.org/10.1111/jrh.12466

[9] Perrin A. Mobile Technology and Home Broadband 2021. [Internet]. Washington DC: Pew Research Center (US). [cited 2021 Jun 3]. Avaialble from: https: //www. pewresearch.org/internet/2021/06/03/mobi le-technology-and-home-broadband-2021/\#fn-27300-1

[10] Lee NT. Public policy can improve older adults' access to techonology. [Internet]. Washington DC: The Brookings Institution (US). 2017 June [cited 2021 June 5]. Available from: https ://www . brookings.edu/blog/techtank/2017/06/05/public-pol icy-can-improve-older-adults-access-to-technology/

[11] MCBS Database [Internet]. 2020 Data user guide: COVID-19 fall supplement public use file. Baltimore (MD): Centers for Medicare \& Medicaid Services (CMS) Office of Enterprise and Data \& Analytis (OEDA) (US). [cited 2021 June 5]. Available 
from: https://www.cms.gov/files/document/2020mcbscov idpuf-dugfall.pdf

[12] MCBS Database [Internet]. Medicare Current beneficiary Survey (MCBS). Baltimore (MD): Centers for Medicare \& Medicaid Services (US). [cited 2021 Jul 29]. Available from: https://www.cms.gov/research-statistics-data-and-s ystems/research/mcbs

[13] Goodman R, Posner S, Huang E, et al. Defining and Measuring Chronic Conditions: Imperatives for Research. Prev Chronic Dis. 2013; 10: E66. PMid: 23618546. https : //doi .org/10.5888/pc d10.120239

[14] Patel SY, Mehrotra A, Huskamp HA, et al. Variation In Telemedicine Use And Outpatient Care During The COVID-19 Pandemic In The United States. Health Aff (Millwood). 2021; 40: 349-58. PMid: 33523745. https://doi.org/10.1377/hlthaff . 2020.01786

[15] Kakani P, Sorensen A, Quinton JK, et al. Patient Characteristics Associated with Telemedicine Use at a Large Academic Health System Before and After COVID-19. J Gen Intern Med. 2021; 36: 1166-8. PMid: 33506387. https://doi.org/10.1007/s11606-020-0 6544-0

[16] Drake C, Lian T, Cameron B, et al. Understanding Telemedicine's "New Normal": Variations in Telemedicine Use by Specialty Line and Patient Demographics. Telemed E Health [Internet]. 2021 March [cited June 5, 2021]. PMid: 33769092. Avaiable from: https: //doi.org/10.1089/tmj.2021.0041

[17] Your Medicare coverage: Telehealth [Internet]. Baltimore (MD): Centers for Medicare \& Medicaid Services (US). [cited June 5, 2021]. Available from: https://www.medicare.gov/coverage/tele health

[18] Medicare Coverage: Virtual check-ins [Internet]. Baltimore (MD): Centers for Medicare \& Medicaid Services (US). [cited June 5, 2021] Available from: https://www.medicare.gov/coverage/virt ual-check-ins

[19] Medicare coverage: E-visits Iinternet]. Baltimore (MD): Centers for Medicare \& Medicaid Services (US). [cited June 5, 2021]. Available from: https://www.medicare.gov/coverage/e-visits
[20] Donaghy E, Atherton H, Hammersley V, et al. Acceptability, benefits, and challenges of video consulting: a qualitative study in primary care. Br J General Prac. 2019; 69: e586-94. PMid: 31160368. https://doi.org/10.3399/bjgp19X704141

[21] Hammersley V, Donaghy E, Parker R, et al. Comparing the content and quality of video, telephone, and face-to-face consultations: a non-randomised, quasi-experimental, exploratory study in UK primary care. Br J General Prac. 2019; 69: e595-604. PMid: 31262846. https://doi.org/10.3399/bjgp19X704573

[22] Roberts ET, Mehrotra A. Assessment of disparities in digital access among Medicare beneficiaries and implications for telemedicine. JAMA Intern Med. 2020; 180: 1386-69. PMid: 32744601. https : //doi.org/10.1001/jamainternmed.2020.2666

[23] Bauerly BC, McCord RF, Hulkower R, et al. Broadband access as a public health issue: the role of law in expanding broadband access and connecting underserved communities for better health outcomes. J Law Med Ethics. 2019; 47(2_suppl): 39-42. PMid: 31298126. https://doi.org/10.1177/1073110519857314

[24] Cross RK, Langenberg P, Regueiro M, et al. A randomized controlled trial of TELEmedicine for patients with inflammatory bowel disease (TELE-IBD). Am J Gastroenterol. 2019; 114: 472-82. PMid: 30410041. https://doi.org/10.1038/s41395-018-0272-8

[25] Su GL, Glass L, Tapper EB, et al. Virtual Consultations Through the $\mathrm{V}$ eterans A dministration SCAN-ECHO Project Improves Survival for Veterans With Liver Disease. Hepatology. 2018; 68: 2317-24. PMid: 29729194. https://doi.org/10.1002/hep. 30074

[26] Shah ED, Amann ST, Karlitz JJ. The time is now: a guide to sustainable telemedicine during COVID-19 and beyond. Am J Gastroenterol 2020; 115: 1371-5. PMid: 32694293. https://doi.org/10.143 09/ajg.0000000000000767

[27] Shah ED, Siegel CA. Systems-based strategies to consider treatment costs in clinical practice. Clin Gastroenterol Hepatol. 2020; 18: 1010-4. PMid: 32092398. https://doi.org/10.1016/j.cg h. 2020.02 .030 